University of Nebraska - Lincoln

DigitalCommons@University of Nebraska - Lincoln

\title{
Transparent vs. Opaque vs. Translucent Wavelength-Routed Optical Networks
}

\author{
Byrav Ramamurthy \\ University of Nebraska-Lincoln, bramamurthy2@unl.edu \\ Helena Feng \\ University of California - Davis \\ Debasish Datta \\ Indian Institute of Technology - Kharagpur \\ Jonathan P. Heritage \\ University of California - Davis \\ Biswanath Mukherjee \\ Indian Institute of Technology - Kharagpur
}

Follow this and additional works at: https://digitalcommons.unl.edu/cseconfwork

Part of the Computer Sciences Commons

Ramamurthy, Byrav; Feng, Helena; Datta, Debasish; Heritage, Jonathan P.; and Mukherjee, Biswanath, "Transparent vs. Opaque vs. Translucent Wavelength-Routed Optical Networks" (1999). CSE Conference and Workshop Papers. 81.

https://digitalcommons.unl.edu/cseconfwork/81

This Article is brought to you for free and open access by the Computer Science and Engineering, Department of at DigitalCommons@University of Nebraska - Lincoln. It has been accepted for inclusion in CSE Conference and Workshop Papers by an authorized administrator of DigitalCommons@University of Nebraska - Lincoln. 


\section{Transparent vs. opaque vs. translucent wavelength-routed optical networks}

Byrav Ramamurthy ${ }^{\odot}$, Helena Feng ${ }^{\dagger}$, Debasish Datta ${ }^{\circledR}$, Jonathan P. Heritage ${ }^{\ddagger}$, and Biswanath Mukherjee ${ }^{\oplus}$

$\odot$ Dept. of Comp. Sci. \& Engg.
University of Nebraska-Lincoln
Lincoln, NE $68588-0115$, U.S.A.

${ }^{\otimes}$ Dept. of E \& E Comm. Engg.

Indian Institute of Technology

Kharagpur, WB 721302, India

$\dagger$ Dept. of Applied Sci. $\ddagger$ Dept. of ECE $\oplus$ Dept. of Comp. Sci.

University of California

Davis, CA 95616, U.S.A.

Correspondence author - Byrav Ramamurthy (E-mail: byrav@cse.unl.edu)

Tel: (402) 472-7791; Fax: (402) 472-7767

1. Introduction: A wavelength-routed optical network consists of multi-wavelength crossconnect switches (XCSs) which are interconnected by optical fibers. Some (or all) crossconnects, referred to as nodes in this paper, are also attached to access stations where data from several end-users could be multiplexed onto a single wavelength division multiplexed (WDM) channel. An access station provides optical-to-electronic $(\mathrm{O} / \mathrm{E})$ conversion and vice versa to interface the optical network with conventional electronic equipment. The access station, at an intermediate node, may also be used (as in this study) for signal regeneration on a lightpath. A new call is admitted into the network if a lightpath (a set of free wavelengths along a given route from source to destination) can be established between the call's source and destination stations. Depending on the number of all-optical fragments in a single lightpath, three different approaches may be employed to operate such a network. These approaches are: transparency, opacity, and translucency (to be explained below). Our study concentrates on evaluating the relative merits of these three approaches in a sample network environment.

Transparency, in the strict sense, implies that the physical medium (an optical WDM channel in our case) should support end-to-end communication of data, independent of bit rates and signal formats. Transparent WDM networks readily allow express signals to bypass extensive electronic signal processing at intermediate nodes. However, the quality of an optical signal degrades as it travels though several optical components along its lightpath from its source to destination. The causes of these degradations include optical-fiber nonlinearities; chromatic and polarization-mode dispersion; noise accumulated due to amplified stimulated emission (ASE) from optical fiber amplifiers (e.g. erbium-doped fiber amplifiers (EDFA)); effects of non-flat gain profile and gain saturation in fiber amplifiers; cross-talk introduced at cross connects; etc. To overcome these impairments, "long-distance" lightpaths may require signal regeneration, at one or more intermediate locations in the network, to "clean up" the signals. Signals are regenerated either through an opto-electronic conversion followed by an electro-optic conversion (as assumed in this study) or entirely in the optical domain. At the other extreme of the spectrum from fully transparent networks are fully opaque networks [1], which include such signal regenerators at every intermediate node along a lightpath. Hence, in opaque networks, a single optical hop of a lightpath never spans more than one physical fiber link in the network.

We propose, in this study, an alternative to both fully transparent and fully opaque networks. We term such networks as translucent networks. In a translucent network, a signal from the source travels through the network "as far as possible" before its quality (say, bit-error rate (BER)) degrades, thereby requiring it to be regenerated at an intermediate node. The same signal could be regenerated several times in the network before it reaches the destination. Note that a single optical hop in a translucent network could span one or more fiber links and may even span the entire source-destination route, under the right conditions. We assume that the network resources available are identical in all three cases. In particular, signal regeneration is achieved in the network by employing a receiver and a transmitter at an intermediate node, without any special hardware [2].

2. Methodology: The interconnected-rings network topology (Fig. 1) used in this study is typical of telecommunication networks. This network consists of 15 nodes with an inter-node distance of $100 \mathrm{~km}$. Each node consists of a single transmitter array and a single receiver array (see Fig. 2). In all the experiments, we assume the following: (a) Poisson call arrivals, (b) exponential call-holding time, (c) uniform distribution of source-destination pair for each call, (d) shortest-path routing of lightpaths, and (e) loss of blocked calls. We distinguish between the ideal cases, when the BER of an incoming call is not computed or used to block 
calls and the non-ideal cases, where the BER of each call is computed in advance. The BER threshold (for the non-ideal cases) was set to $10^{-12}$, and one million call requests were simulated. We employ the First-Fit wavelength-assignment algorithm where the first available wavelength in a predetermined order is used to set up a call. For the non-ideal cases, in order to study the impact of switch crosstalk, we vary the parameter $X_{s w}$ (switch crosstalk ratio). Other parameters used in this study are shown in Table 1 and these are used in estimating the BER following the approach described in [3].

For the transparent case, for each incoming call, the BER of a lightpath is estimated before setting up the call. The BER calculations include the effects of fiber loss, crosstalk from crossconnect switches (XCS), ASE from EDFA, and gain saturation at EDFA. Also taken into account are the effects of other ongoing traffic in the network. Optical fiber nonlinearities and dispersion are ignored in this study, although these parameters are currently being investigated.

For the opaque case, the signal is regenerated at the end of each physical link. It is assumed the transmission over a single physical link does not seriously degrade the quality of the signal, under the given system parameters. Hence, the results for the opaque case are identical irrespective of whether the BER of calls are employed to block calls or not.

The translucent case is similar to the transparent case in that the BER is first estimated on an available single-hop lightpath. However, if that lightpath is unavailable (either because of unavailable resources or because the BER turns out to be above the acceptable limit), then the lightpath is broken into two fragments (at the node half-way [4] along the lightpath) and a free receiver-transmitter pair, if available, at the half-way node is employed for regeneration. The call is accepted only if both the fragments can be established in the network with adequate BER. Currently, the new lightpaths are not broken up into smaller fragments, but this is also a direction of our ongoing investigation.

3. Results and Discussion: The results of the simulation experiments are shown in Figures 3 and 4 . Figure 3 shows the network performance when the near-ideal physical layer parameters [5] are used (with $X_{s w}=-30 \mathrm{~dB}$ ). For a wide range of loads, we find that using the opaque approach results in a far greater number of blocked calls compared to the other two approaches, viz., transparent and translucent. This is because of the fact that the opaque approach uses a greater number of transmitters and receivers to set up a single lightpath compared to the other two approaches. Further, we note that the translucent approach performs a little better than the transparent approach. This can be explained by the fact that the transparent approach requires a single free wavelength along the entire source-destination route in order to establish a lightpath and this may be difficult to find for long routes in the network. The translucent approach, on the other hand, provides a second chance to the call by breaking it into two fragments, if necessary. The results indicate that the use of the receiver-transmitter pair at the intermediate node, to regenerate the signal, is well justified in the translucent case.

When the crosstalk in the network increases (as a result of the switch crosstalk ratio, $X_{s w}$, increasing to $-25 \mathrm{~dB}$ ), we find that blocking increases for both the transparent and translucent approaches, unlike for the opaque approach when it remains unchanged (see Fig. 4). Moreover, we find that the translucent approach performs much better than the transparent approach in this case. This can be explained by the fact that with increased system impairments, the BER of a call increases and this is aggravated in case of long-distance calls which must traverse several crossconnect switches. Hence, it is a good idea to allow calls to be broken into shorter fragments, using the translucent approach.

Additional receivers and transmitters at each intermediate node, to be exclusively used for signal regeneration, would benefit both the opaque and translucent approaches, to varying degrees. Both the opaque and translucent approaches need a pair of electronic switches at the intermediate nodes, which results in higher implementation costs.

4. Conclusion: There has been a great deal of discussion regarding transparency vs. opacity in (nationalscale) optical wavelength division multiplexed (WDM) networks [1]. Our study shows that, for medium-scale networks, where physical-layer characteristics are dominated by crosstalk and ASE, translucency can help to improve the overall network performance. For larger-scale networks, where impairments introduced by fiber nonlinearities and dispersion cannot be ignored, we anticipate that a higher degree of opacity may be needed to combat signal degradations, but this is an open problem for further research.

\section{References}

[1] E. Goldstein, J. Nagel, J. Strand, and R. Tkach. National-scale networks likely to be opaque. Lightwave Xtra!, Feb. 1998. 
[2] However, note the use of a pair of electronic switches (marked ESW in Fig. 2) in this architecture.

[3] D. Datta, B. Ramamurthy, H. Feng, J. P. Heritage, and B. Mukherjee. BER-based call admission in wavelength-routed optical networks. In Optical Fiber Communication (OFC '98) Technical Digest, San Jose, CA, Feb. 1998.

[4] The half-way node is chosen using the number of fiber links on the source-destination route; in case of a tie, the one closer to the source is chosen.

[5] The results obtained for the ideal cases are almost identical to those obtained with near-ideal physical layer parameters and using BER (see Fig. 3), and hence are not shown.

\begin{tabular}{||l|l||}
\hline \hline Parameter & Value \\
\hline \hline Number of wavelengths & 8 \\
\hline Wavelength-spacing & $100 \mathrm{GHz}$ \\
\hline Wavelengths (in $\mathrm{nm})$ & $1547-1553$ \\
\hline Bit rate per channel $(r)$ & $1 \mathrm{Gbps}$ \\
\hline Electronic bandwidth $\left(B_{e}\right)$ & $0.7 r$ \\
\hline Multiplexer loss $\left(L_{m x}\right)$ & $4 \mathrm{~dB}$ \\
\hline Demultiplexer loss $\left(L_{d m}\right)$ & $4 \mathrm{~dB}$ \\
\hline Switch loss $\left(L_{s w}\right) 4 \times 4$ switch & $8 \mathrm{~dB}$ \\
\hline
\end{tabular}

\begin{tabular}{|||l|l||}
\hline \hline Parameter & Value \\
\hline \hline Tap loss $\left(L_{\text {tap }}\right)$ & $1 \mathrm{~dB}$ \\
\hline Fiber loss $\left(L_{f}\right)$ & $0.2 \mathrm{~dB} / \mathrm{km}$ \\
\hline Input EDFA Gain $\left(G_{i n}\right)$ & $22 \mathrm{~dB}$ \\
\hline Output EDFA Gain $\left(G_{o u t}\right)$ & $16-18 \mathrm{~dB}$ \\
\hline ASE factor $\left(n_{s p}\right)$ & 1.5 \\
\hline Max. laser power $\left(P_{t}\right)$ & $1 \mathrm{~mW}$ \\
\hline Switch crosstalk ratio $\left(X_{s w}\right)$ & $-30 \mathrm{~dB},-25 \mathrm{~dB}$ \\
\hline Excess small-signal gain $(d G)$ & $3 \mathrm{~dB}$ \\
\hline
\end{tabular}

Table 1: System parameters and their values used in this study.

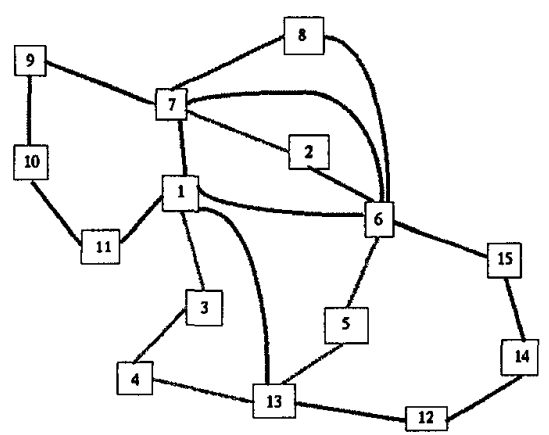

Figure 1: The interconnected-rings network used in this study. Each inter-node link is $100 \mathrm{~km}$ long.

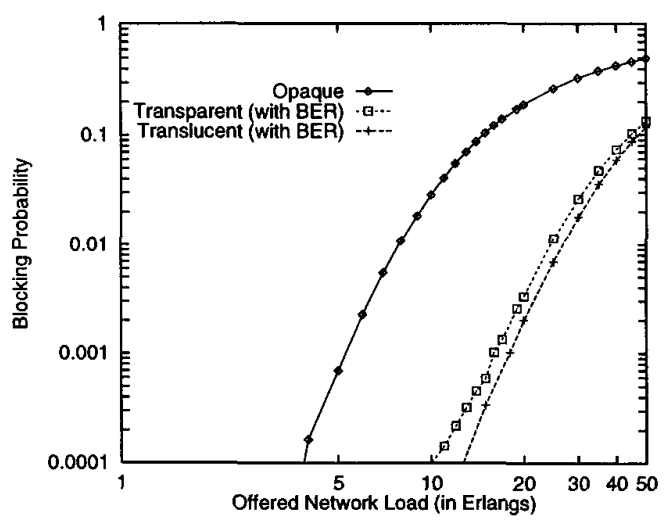

Figure 3: Blocking probability vs. network-wide load when $X_{s w}=-30 \mathrm{~dB}$.

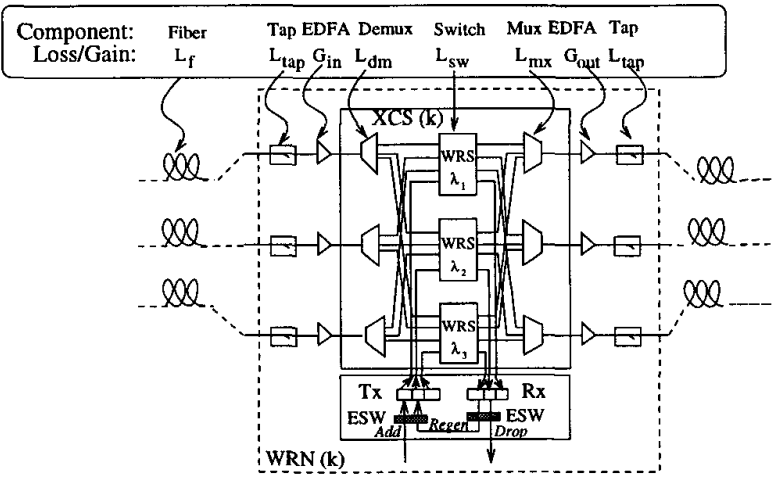

Figure 2: A node in the interconnected-rings network used in this study.

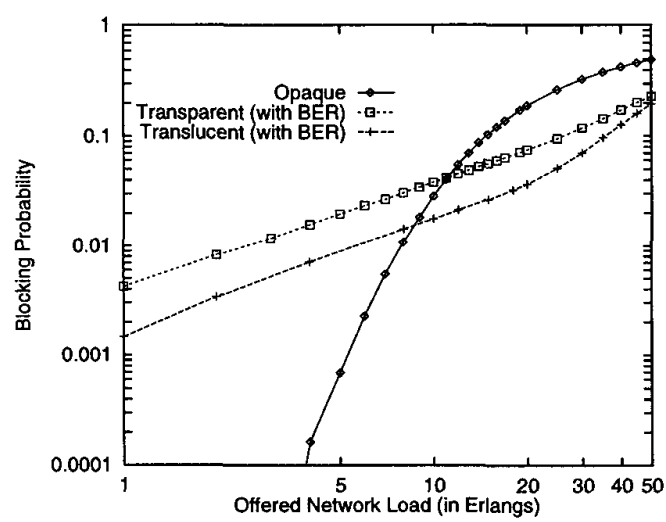

Figure 4: Blocking probability vs. network-wide load when $X_{s w}=-25 \mathrm{~dB}$. 